\title{
The enzymic hydrolysis and structure of phosphatidyl glycerol
}

Phosphatidyl glycerol (I) was recognized by Benson And MARUo', 2 to be a major phospholipid of photosynthetic tissues and its structure was ascertained by chemical degradation. This polyglycerol phospholipid is also abundant in various species of bacteria $^{3-5}$ and is distributed in a low concentration in various animal tissues. As far as the authors are aware, no data are available about the enzymic hydrolysis of phosphatidyl glycerol. A study in this direction was undertaken in order to outline possible catabolic pathways of this phospholipid and to confirm its chemical structure along an independent route.

In these preliminary studies use was made of micro quantities of pure $\left[{ }^{32} \mathrm{P}\right]-$ phosphatidyl glycerol isolated from Bacillus cereus and Bacillus megatherium cultured for $\mathrm{I} 6 \mathrm{~h}$ in a broth containing radioactive orthophosphate. The harvested bacteria were extracted according to the method of BLIGH AND DYER ${ }^{6}$ and the crude lipids fractionated on a column of silica acid (Mallinckrodt) with chloroform-methanol mixtures as eluents. The radioactivity of the effluent was scanned by an automatic device. Neutral lipids were eluated by chloroform while cardiolipin (and closely related phospholipids), together with phosphatidic acid, were removed by prolonged elution with chloroform - methanol $(98: 2, \mathrm{v} / \mathrm{v})$. The main portion of phosphatidyl glycerol was recovered in the chloroform - methanol $(94: 6, \mathrm{v} / \mathrm{v})$ effluent. Occasionally this fraction was contaminated with phosphatidyl ethanolamine; however, rechromatography of such fractions under similar conditions effected a complete separation of both phospholipids.

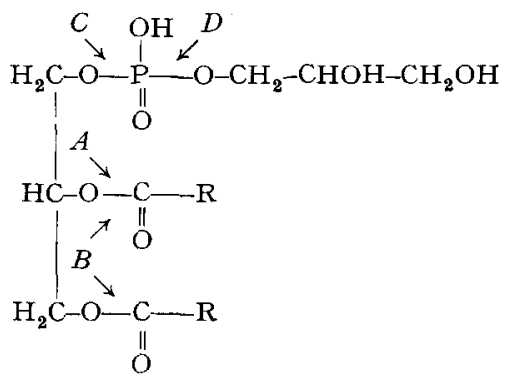

(I)

Scheme I. Formula of phosphatidyl glycerol as ascertained by Benson and Maruo ${ }^{2}$. The arrows indicate the site of attack of phospholipase A, B, C and D, respectively, as established in the present study.

The isolated phosphatidyl glycerol revealed upon chromatography on silicaimpregnated paper ${ }^{7}$ only one radioactive zone (Fig. I), coinciding with a green spot developed by staining with the tricomplex procedure of BUNGENBERG DE JONG ${ }^{8}$. Thin-layer chromatograms ${ }^{9}$ developed in various systems gave only one spot after treatment with $\mathrm{H}_{2} \mathrm{SO}_{4}$. Autoradiograms of two-dimensional paper chromatograms (phenol-water; butanol-propionic acid-water) prepared of the water-soluble phosphodiester obtained by mild alkaline hydrolysis of phosphatidyl glycerol, revealed the radioactive compound to be located in a position characteristic for glycerylphosphoryl glycerol. 
Phosphatidyl glycerol was subjected to hydrolysis by phospholipase A, B, C and $\mathrm{D}$, which enzymes have an established mode of action towards well-known phospholipids such as lecithins and cephalins. Phospholipase A (phosphatide acylhydrolase, EC 3.I.I.4) from snake venom (Crotalus adamantheus) was found to hydrolyse phosphatidyl glycerol in a Tris-buffered system ( $\mathrm{pH}$ 7.I) after an incubation of $2 \mathrm{~h}$ (Fig. I) The chromatographic behaviour of the radioactive hydrolysis product indicated that by action of phospholipase $A$, as in the case of lecithin, one fatty acid is liberated, and a lyso-phosphatidyl glycerol is produced. Actually, thin-layer chromatograms demonstrated the presence of free fatty acid in the enzymic hydrolysate.

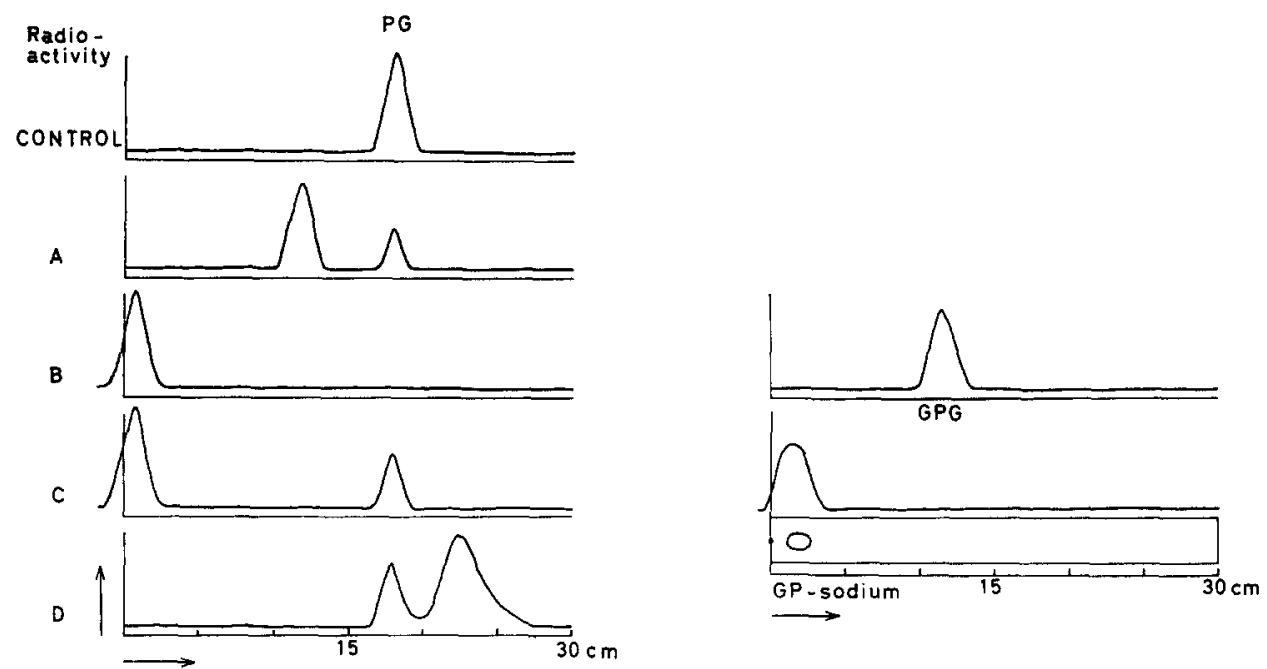

Fig. I. Paper-chromatographic demonstration of the enzymic hydrolysis of phosphatidyl glycerol. Ordinate, radioactivity scanned on paper chromatogram; abscissa, position on paper chromatograms. Left hand, silica-impregnated paper chromatograms of purified [ ${ }^{32} \mathrm{P}$ phosphatidyl glycerol (control) and of solutions after action of phospholipase A, B, C and D, respectively. Right hand: paper chromatograms developed on Whatman paper No. I with phenol-water showing, after action of phospholipase $\mathrm{B}$ and $\mathrm{C}$, as water-soluble products, glycerylphosphoryl glycerol (GPG) and glycerophosphate (GP), respectively.

Phospholipase B (lysolecithin acyl-hydrolase EC 3.I.I.5) from rat spleen ${ }^{10}$ acted under similar conditions as phospholipase A, on both phosphatidyl glycerol (Fig. I) and on the lyso-phosphatidyl glycerol produced by the snake venom. The radioactive hydrolysis product formed was water-soluble and was shown to be identical with the deacylated derivative viz. glycerylphosphoryl glycerol.

Phospholipase C (phosphatidylcholine cholinephosphohydrolase EC 3.r.4.3) from Clostridium welchii was rather ineffective towards phosphatidyl glycerol, but the corresponding enzyme from $B$, cereus, known to act more readily on negatively charged phospholipids, did give in a Tris-buffered medium $\left(\mathrm{pH}_{7}\right)$ a breakdown of phosphatidyl glycerol (Fig. I). The water-soluble radioactive hydrolysis product was on paper chromatograms in five solvent systems indistinguishable from glycerophosphate. Confirmatory, thin-layer chromatograms ${ }^{9}$ demonstrated the formation of diglycerides.

In an aqueous-ethereal system phosphatidyl glycerol was susceptible to the action of phospholipase D (phosphatidylcholine phosphatidohydrolase EC 3.I.4.4.) obtairied 
from Brussels sprouts ${ }^{11}$. The paper chromatogram given in Fig. I shows the breakdown after an incubation of $30 \mathrm{~min}$. After a 2-h incubation phosphatidyl glycerol was completely converted into a radioactive compound chromatographically identical with phosphatidic acid obtained either by chemical synthesis or by phospholipase D action on ${ }^{32} \mathrm{P}-$ labelled yeast lecithin. In agreement free glycerol was recovered in the hydrolysate.

As summarized in Scheme I, phosphatidyl glycerol is susceptible to the action of phospholipases $\mathrm{A}, \mathrm{B}, \mathrm{C}$ and $\mathrm{D}$, the results of the enzymic hydrolysis allowing a number of conclusions supporting the structure (I) as assignated by BENSON AND MARUo to this phospholipid Inasmuch as phospholipase A is inactive towards D- $\alpha$ phospholipids and removes from $\mathrm{L}-\alpha$-compounds the $\beta$-fatty acids only, the results obtained suggest phosphatidyl glycerol to have the $\mathrm{L}-\alpha$-configuration. Some caution is necessary, however, since in the authors' laboratory it was recently demonstrated that synthetic $\beta$-phospholipids can also be hydrolyzed by phospholipase A. The results with phospholipase $B$ showed glycerylphosphoryl glycerol to be part of the phosphatidyl glycerol molecule. Inasmuch as phospholipase C produces glycerophosphate and diglycerides, one glycerol moiety of phosphatidyl glycerol is apparently not esterified with fatty acid chains. The formation of phosphatidic acid and glycerol by phospholipase $\mathrm{D}$ confirms the presence in phosphatidyl glycerol of a diacylglycerylphosphoric acid unit linked with glycerol.

Further studies are in progress to investigate the asymmetry of the glycerol moiety ${ }^{12}$ and the configuration of phosphatidyl glycerol on account of the nature and configuration of enzymic hydrolysis products obtained on a preparative scale.

This investigation is supported by the Netherlands Organization for the Advancement of Pure Research. The authors thank Mr. G. L. ScherphoF and Mr. Th. Wensing for the collaboration in the isolation of various enzymes.

$\begin{array}{cl}\text { Department of Biochemistry, } & \text { F. HAVERKATE } \\ \text { Laboratory of Organic Chemistry, State University, } & \text { U. M. T. HoUTSMULLER } \\ \text { Utrecht (The Netherlands) } & \text { L. L. M. VAN DEENEN }\end{array}$

1 B. Maruo and A. A. Benson, J. Am. Chem. Soc., 79 (1957) 4564 .

2 A. A. Benson and B. Maruo, Biochim. Biophys, Acta, 27 (1958) I89.

3 M. G. MacFarlane, Biochem. $J$., 8o (I96I) 45 P.

4 M. Kates, D. J. Kushner and A. T. James, Can. J. Biochem. Physiol, 40 (1962) 83.

5 L. L. M. Van Deenen, U. M. T. Houtsmuller and F. Haverkate, Proc. Deuel Conf. on Lipids, 1962 , in the press.

${ }^{6}$ E. G. Bligh and W. J. Dyer, Can. J. Biochem. Physiol., 37 (I959) 9 I I.

7 G. V. Marinetti, J. Erbland and J. Kochen, Federation Proc., 16 (I957) 837.

${ }^{8}$ H. G. Bungenberg De Jong, Koninkl. Ned. Akad. Wetenschap. Proc. B, 64 (196I) 583.

9 J. J. PeIfer, Mikrochim. Acta, (I962) 529.

10 E. A. Marples and R. H. S. Thompson, Biochem. J., 74 (I960) I23.

11 F. M. Davidson and C. Long, Biochem. $J$., 69 (I958) 458.

12 A. A. Benson and M. MiYano, Biochem. $J$., 8I (I96I) 3 I P.

Received July r2th, r962 